\title{
New Wide Field J- and K-band Galaxy Counts and the Extragalactic Background
}

\author{
P. Väisänen
}

Helsinki University Observatory, Helsinki 00014, Finland

E. V. Tollestrup \& S. P. Willner

Harvard-Smithsonian Center for Astrophysics, Cambridge, MA 02138, USA

\author{
Martin Cohen \\ Radio Astronomy Laboratory, 601 Campbell Hall, University of \\ California, Berkeley, CA 94720, USA
}

\begin{abstract}
We present new medium-deep wide field galaxy counts at $J$ - and $K$-bands, performed in the ELAIS fields. The source detection limits are at $J=19.5$ and $K=18.0$ magnitudes. The sky coverage is 1 degree $^{2}$, making our survey the largest to date at these wavelengths and magnitudes. Counts in this brightness range are critical for normalization of models when explaining the deepest galaxy counts. In our survey fields we find evidence for a higher than expected local galaxy density. If normalized to our counts, galaxy models do not need strong brightness evolution or exotic populations to be explained even at the faintest levels. We measure clearly sub-Euclidean slopes of $d \log N(m) / d m \sim 0.45$ to 0.50 at $13<K<17.5$ and $14.5<J<19$. Normal, passively evolving stellar populations along with a high normalization of the local luminosity function and an open cosmological model best fit both the slope and amplitude of our number counts. We report a preliminary value, $\sim 30 \mathrm{nWm}^{-2} \mathrm{sr}^{-1}$, for the $K$-band extragalactic background light using zodi-subtracted DIRBE-data and our observed star counts. The value is consistent with, e.g., Matsumoto (these Proceedings 2001) and Gorjian, V., Wright, E. L., \& Chary, R. R. 2000, ApJ, 536, 550, but is considerably higher than extrapolations from faint galaxy counts.
\end{abstract}

\section{Discussion}

Gerhardt Meurer: How does your new EBL affect the claim by Pozzetti et al. that there cannot be high extinction at high $z$ because it would overproduce the local $K$-band light?

Petri Väisänen: As discussed in this meeting, I think it is important to first sort out the uncertainties in zodiacal-light models used in deriving these EBL values and also to look into galaxy-photometry issues related to deriving lower limits to EBL from galaxy counts. If the high observational EBL values 
hold up, and current understanding of high- $z$ extinction and metallicity do not significantly change, other sources than galaxies might have to be considered for part of the EBL. However, some recent galaxy evolution models (Jimenez \& Kashlinsky 1999, ApJ, 511, 16) are able to produce the high optical-NIR EBL. 